\title{
Linear Dispersion Codes
}

\author{
Babak Hassibi ${ }^{1}$ \\ Dept. of Electrical Engineering \\ California Institute of Technolog \\ Pasadena, CA 91125 \\ e-mail: \\ hassibi@systems.caltech.edu
}

\author{
Bertrand Hochwald \\ Lucent Technologies \\ 600 Mountain Avenue, Rm. 2C-363 \\ Murray Hill, NJ 07974 \\ e-mail: hochwald@bell-labs.com
}

\begin{abstract}
Multiple-antenna systems that operate at high rates require simple yet effective space-time transmission schemes to handle the large traffic volume in real time. At rates of tens of bits $/ \mathrm{sec} / \mathrm{Hz}$, V-BLAST, where every antenna transmits its own independent substream of data, has been shown to have good performance and simple encoding and decoding. Yet V-BLAST suffers from its inability to work with fewer receive antennas than transmit antennas-this deficiency is especially important for modern cellular systems where a basestation typically has more antennas than the mobile handsets. Furthermore, because V-BLAST transmits independent data streams on its antennas there is no built-in spatial coding to guard against deep fades from any given transmit antenna. On the other hand, there are many previouslyproposed space-time codes that have good fading resistance and simple decoding, but these codes generally have poor performance at high data rates or with many antennas.
\end{abstract}

We propose a high-rate coding scheme that can handle any configuration of transmit and receive antennas and that subsumes both V-BLAST and many proposed space-time block codes as special cases. The scheme transmits substreams of data in linear combinations over space and time. The codes are designed to optimize the mutual information between the transmitted and received signals. Because of their linear structure, the codes retain the decoding simplicity of V-BLAST, and because of their informationtheoretic optimality, they possess many coding advantages. We give examples of the codes and show that their performance is generally superior to earlier proposed methods over a wide range of rates and SNR's.

A full version of this paper may be downloaded from the web site http://mars.bell-labs.com

An example of a linear dispersion code we obtain for three antennas is
Figure 1 compares the performance of an orthogonal design [1] for three antennas with the linear dispersion code for rate six bits/channel use. We achieve this rate by having the orthogonal design use a 256-QAM constellation, and the linear dispersion code use 64-QAM.

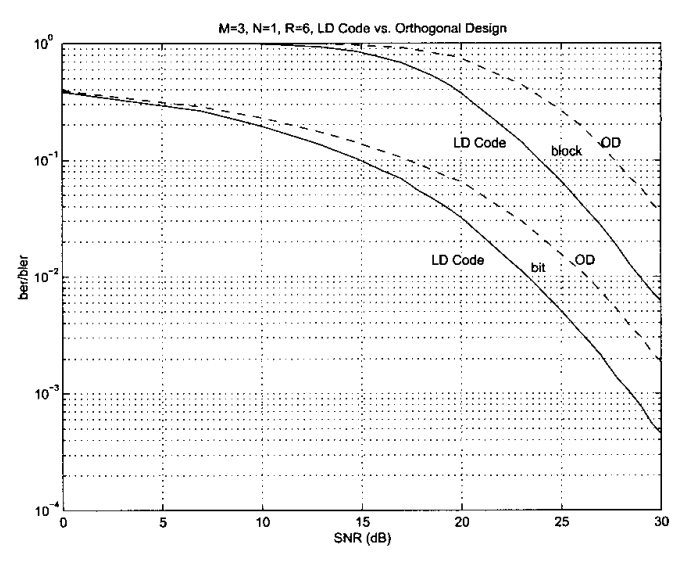

Figure 1: Block and bit error performance of orthogonal design and the linear dispersion code for three antennas. The rate is six bits/channel use, obtained in the orthogonal design by transmitting 256-QAM and obtained in the linear dispersion code by transmitting 64-QAM on $s_{1}, \ldots, s_{4}$. The decoding in both cases is nulling/cancelling. The comparison of block error is meaningful here because the block size in both cases is four time samples.

\section{REFERENCES}

[1] V. Tarokh, H. Jafarkhani and A. R. Calderbank, "Space-time block codes from orthogonal designs," IEEE Trans. Info. Theory., vol. 45, pp. 1456-1467, July 1999.

$S=\left[\begin{array}{ccc}\alpha_{1}+\alpha_{3}+j\left[\frac{\beta_{2}+\beta_{3}}{\sqrt{2}}+\beta_{4}\right] & \frac{\alpha_{2}-\alpha_{4}}{\sqrt{2}}-j\left[\frac{\beta_{1}}{\sqrt{2}}+\frac{\beta_{2}-\beta_{3}}{2}\right] & 0 \\ \frac{-\alpha_{2}+\alpha_{4}}{\sqrt{2}}-j\left[\frac{\beta_{1}}{\sqrt{2}}+\frac{\beta_{2}-\beta_{3}}{2}\right] & \alpha_{1}-j \frac{\beta_{2}+\beta_{3}}{\sqrt{2}} & -\frac{\alpha_{2}+\alpha_{4}}{\sqrt{2}}+j\left[\frac{\beta_{1}}{\sqrt{2}}-\frac{\beta_{2}-\beta_{3}}{2}\right] \\ 0 & \frac{\alpha_{2}+\alpha_{4}}{\sqrt{2}}+j\left[\frac{\beta_{1}}{\sqrt{2}}-\frac{\beta_{2}-\beta_{3}}{2}\right] & \alpha_{1}-\alpha_{3}+j\left[\frac{\beta_{2}+\beta_{3}}{\sqrt{2}}-\beta_{4}\right] \\ \frac{\alpha_{2}-\alpha_{4}}{\sqrt{2}}+j\left[\frac{\beta_{1}}{\sqrt{2}}+\frac{\beta_{2}-\beta_{3}}{2}\right] & -\alpha_{3}+j \beta_{4} & -\frac{\alpha_{2}+\alpha_{4}}{\sqrt{2}}+j\left[\frac{\beta_{1}}{\sqrt{2}}-\frac{\beta_{2}-\beta_{3}}{2}\right]\end{array}\right]$.

where $s_{j}=\alpha_{j}+i \beta_{j}, j=1, \ldots, 4$ are taken from a QAM constellation, and the three columns of $S$ represent the symbols transmitted from each of the three antennas as functions of time. This code has a mutual information of $6.25 \mathrm{bits} / \mathrm{channel}$ use at $\mathrm{SNR}=20 \mathrm{~dB}$, which is most of the channel capacity.

\footnotetext{
${ }^{1}$ Professor Hassibi left Lucent Technologies and joined Caltech in January.
} 